\title{
Scientific Poster Presentation Abstracts
}

${ }^{*}$ Corresponding author: Tabriz University of Medical Sciences, Tabriz, Iran.Email:nazila.moftian@gmail.com

\begin{abstract}
Background:Scoliosis is a three-dimensional deformity of the spine that is commonly assessed through measuring the Cobb angle.

Objectives: In this study, a Cobb angle measurement decision support system (CaMDSS) was presented to provide a repeatable and reproducible procedure for Cobb angle measurement in idiopathic scoliosis patients.
\end{abstract}

Methods: We used the OpenCV and the Numpy library for image processing and system design. A series of 98 anterior-posterior radiographs from patients diagnosed with idiopathic scoliosis were used to assess the repeatability and reproducibility of CaMDSS. Five independent observers performed the measurements, and each image was analyzed by each observer three times with a minimum interval of two weeks between measurements. Both the intra- and inter-observer reliability were obtained using the single measure intraclass correlation coefficient (ICC) value. The mean absolute difference (MAD) and the standard error measurement (SEM) were calculated for all corresponding intra- and inter-observer reliability estimates.

Results: Statistical results for the inter-observer analysis showed that the MAD between manual and CaMDSS was less than $3^{\circ}$, and the ICCs ranged from 0.94 to 0.99 . The combined SEM between all five observers for intra-observer measurements of the manual method and CaMDSS was $1.79^{\circ}$ and $1.27^{\circ}$, respectively. The inter-observer reliability of CaMDSS was excellent as the ICC value of 0.97 with $95 \%$ CI was obtained. The CaMDSS mean absolute difference was $2.18 \pm 2.01$ degrees.

Conclusion: Our study showed CaMDSS was an efficient and reliable method to assess the scoliotic curvature in Thoraco-Lumbar standing radiographs with the possibility of expediting clinic visits, ensuring the reliability of calculation, and decreasing the patient's exposure to radiation.

Keywords:Cobb Angle; Decision Support System; Scoliosis; Spine Deformity; Radiography; Image Processing

\section{-10.5812/IRANJRADIOL.99152}

\section{Designing a Structured System for Mammography Reporting}

Sedigheh Emadi ${ }^{1}$; Sina Kardeh ${ }^{1}$; Sepideh Sefidbakht ${ }^{2}$; Alireza Shakibafard ${ }^{2}$; Omid Pournik ${ }^{3}$; Roxana Sharifian ${ }^{1, *}$
${ }^{1}$ Department of Management and Medical Information Sciences, Shiraz University of Medical Sciences, Shiraz, Iran

${ }^{2}$ Department of Radiology, Medical Imaging Research Center, Shiraz University of Medical Sciences, Shiraz, Iran

${ }^{3}$ Department of Community Medicine, School of Medicine, Iran University of Medical Sciences, Tehran, Iran

*Corresponding author: Department of Management and Medical Information Sciences, Shiraz University of Medical Sciences, Shiraz, Iran. Email: sharifianr@sums.ac.ir

\section{Abstract}

Background: Breast cancer is among the top causes of cancer-related mortality among females in both developed and developing countries. Screening for breast cancer plays a crucial role in the prevention of disease burden. Among various imaging modalities, mammography is currently considered the first step for the detection of breast lesions. The mammography data of breast cancer patients encompass a wide array of texts related to specific visual findings. Accordingly, unorganized mammography documents usually impede physicians' efforts to fully review the previous information in follow-up visits; hence, this not only affects clinical judgment and treatment planning adversely, but also adds to the financial burdens and workload imposed on the healthcare system. This further highlights the great potential of structured mammography reporting in the management of breast cancer patients.

Methods: In the first step, an expert panel consisting of two attending radiologists and a health informatician (all affiliated to the Shiraz University of Medical Sciences, Shiraz, Iran) reviewed related guidelines to determine the appropriate items, ontologies, and standard formats for data entry. These included radiology textbooks and the systematized nomenclature of medicine (SNOMED). In addition, 100 mammography reports were completely examined for data extraction and the creation of a template report. In the next step, the structure of the data registry was discussed among a group of radiologists and breast cancer surgeons during several sessions using Delphi technique.

Results: Overall, 119 fields were selected for data entry. Our survey showed that all of the contributing physicians believed that a structured reporting system for mammography can help standardize and reduce reporting time and errors. Following the completion of the template, a user interface was developed by the expert panel for integration in the cloud software workflow, 


\section{SCIENTIFIC Poster Presentation Abstracts}

which will be deployed and assessed in the next phase of the project.

Conclusion: Structured mammography reporting helps radiologists and surgeons to efficiently and confidently track the management course of their patients. In addition, with the advancement of artificial intelligence, especially deep learning for image classification, clean and labeled image databases can be used for designing computer-assisted decision support systems without significant data preprocessing.

Keywords: Structured Reporting; Mammography; Breast Cancer; Radiology; Artificial Intelligence

\section{• 10.5812/IRANJRADIOL.99153}

\section{Using Kalman Filter to Improve the Accuracy of Diffusion Coefficients in MR Imaging: A Simulation Study}

Sam Sharifzadeh Javidi1, 2, *; Hamidreza Saligheh Rad ${ }^{1,2}$

${ }^{1}$ Physics and Medical Engineering Department, Medicine School, Tehran University of Medical Sciences, Tehran, Iran

${ }^{2}$ Quantitative Medical Imaging Systems Group, Research Center for Molecular and Cellular Imaging, Tehran University of Medical Sciences, Tehran, Iran

${ }^{*}$ Corresponding author: Physics and Medical Engineering Department, Medicine School, Tehran University of Medical Sciences, Tehran, Iran.Email: samjavidi@yahoo.com

\section{Abstract}

Background: The diffusion coefficient of water molecules in different tissues is a biomarker to diagnose and differentiate diseases, tumors, injuries, etc. [1]. Using a motion-sensitizing gradient, it is possible to map the diffusion coefficient into diffusionweighted MRI (DWI) [2]. Although DWI is a good tool for diagnosing, its accuracy in low regime SNR and even especially in high blood consumer organs is in question.

Objectives: We aimed to improve the accuracy of the diffusion coefficient, especially in the presence of noise.

Methods: Diffusion of water molecules at each voxel causes a signal intensity decay that can be measured using a motion-sensitizing gradient. Blood perfusion in capillary network artifacts and measurement noise can affect the real amount of D. In light of considering process noise and measurement noise, Kalman filter is used to cancel perfusion artifacts and measurement noise. Based on the diffusion model ( $S=S 0 \exp (-b D))$, signal intensity was produced several times and a complex Gaussian noise was added to it. Using a Kalman filter, a noise cancelation process was designed to improve the quality of results. The Kalman filter solved a linear problem in the form of state space. Therefore, the diffusion model was rewritten as $\log (\mathrm{S} / \mathrm{SO}) /-\mathrm{b}=\mathrm{D}$ in state space. The Kalman filter predicted the amount of $\mathrm{D}$ and then modified it based on measurements iteratively (Figure 1). Finally, the results of the proposed method and conventional method were compared with true values (Figure 2).

Results: Statistical tests showed that the proposed method was significantly better than the conventional method $(\mathrm{P}<0.01)$. The conventional method caused a bias in the results of DWI due to eliminating micro-vessel perfusion in the capillary network. However, Kalman filter could consider the effects of microvessel perfusion as a process noise and reduce its effects on results. Kalman filter results (Table 1) were compared with true values and the t-test showed no significant alteration ( $P$ $=0.25$ ). Conventional method results were significantly different from true values and Kalman filter results $(\mathrm{P}<0.0001)$.

Conclusion: Diffusion coefficients in the presence of noise and capillary network suffer from bias; however, the proposed method can be used in these situations to improve the quality of DWI images.

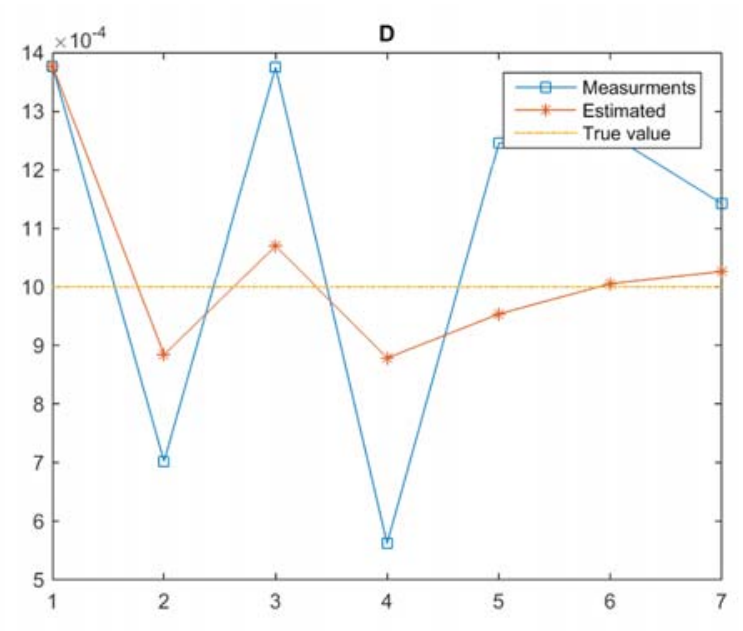

Figure 1. Measurement of D is shown as blue color and varies because of noise, although; Kalman filter improves the result and increases accuracy. 